\title{
Association between the rs615563 variant of PCSK9 gene and circulating lipids and Type 2 diabetes
}

\author{
Samira Taghizadeh Jazdani ${ }^{1,2}$, Hajieh Bibi Shahbazian ${ }^{1}$, Bahman Cheraghian ${ }^{3}$, Mohammad Taha Jalali ${ }^{2,4}$ and \\ Narges Mohammadtaghvaei ${ }^{2,4^{*}}$ (i)
}

\begin{abstract}
Objective: Many different genetic variants of proprotein convertase subtilisin kexin 9 (PCSK9) are related to the serum levels of cholesterol and LDL cholesterol (LDL-C). The rs615563 variant of PCSK9 (a gain-of-function mutation) is associated with increased triglycerides and cholesterol levels, but its association with the incidence of diabetes is not well defined. This study aimed to investigate the relationship between the PCSK9 rs615563 variant with the incidence of type 2 diabetes. The data reported in this study are based on subsamples from a 5-year (2009-2014) cohort study of the adult population (590 subjects) aged 20 years and older. The rs615563 polymorphism was genotyped using polymerase chain reaction (PCR) followed by restriction fragment length polymorphism (RFLP) analysis.

Results: The distribution of PCSK9 rs615563 genotypes was not significantly different between the diabetic and nondiabetic individuals. The incidence of diabetes after five-years of follow-up was not different between the genotypes. Our findings also showed no significant relationship between this polymorphism and serum lipid parameters. The data extracted from our cohort study do not support the findings that the gain-of-function mutations of PCSK9 predispose to the incidence of type 2 diabetes.
\end{abstract}

Keywords: Type 2 diabetes, LDL cholesterol, rs615563, PCSK9, PCR-RFLP

\section{Introduction}

The ninth member of the proprotein convertase subtilisin kexin (PCSK9) family was identified in early 2003. PCSK9 gene, also known as neural apoptosis-regulated convertase 1 (NARC1), is located on chromosome 1p32.3. This gene encodes an autocatalytic enzyme predominantly expressed in hepatocytes and is released directly into the circulation. Extrahepatic tissues such as the intestine, kidney, brain, blood vessels, and pancreas express PCSK9 to a lesser extent $[1,30]$.

\footnotetext{
*Correspondence: ntaghvaie@gmail.com

${ }^{2}$ Department of Laboratory Sciences, Faculty of Paramedicine, Ahvaz

Jundishapur University of Medical Sciences, Ahvaz, Iran

Full list of author information is available at the end of the article
}

PCSK9 has a critical role in the regulation of low-density lipoprotein cholesterol levels. PCSK9 binds to the hepatic LDL receptors and promotes their degradation, leading to decreased number of LDL receptors on hepatocytes [12]. Several different genetic variants of PCSK9 have been reported in various populations, including gain-of-function (GOF) and loss-of-function (LOF) variants $[6,8,11,19,20]$. Gain-of-function PCSK9 variants can increase plasma LDL cholesterol levels by accelerating LDL receptors degradation, and in contrast, loss-offunction variants of PCSK9 can decrease plasma LDL cholesterol levels by reducing LDL receptors degradation [16]. PCSK9 is known as a therapeutic target to control LDL cholesterol levels due to the significant role of PCSK9 on plasma LDL cholesterol clearance [34]. The relationship between plasma PCSK9 concentration and 
diabetes mellitus has been of note in some previous studies. The majority of the results showed that diabetes was significantly associated with increased plasma levels of PCSK9 [7, 21, 23], but there are also contradictory results showing an inverse relationship [5, 9]. This controversy may be explained by the fact that PCSK9 is also expressed in the pancreas, and this locally produced PCSK9 may play the main role in insulin secretion impairment. In the PCSK9 deficiency condition, although the plasma cholesterol level is decreased, cholesterol accumulation in the $\beta$-cells increases, leading to $\beta$-cell dysfunction and diabetes [13].

Genetic studies have shown that PCSK9 alleles associated with reduced LDL cholesterol levels increase the risk of developing type 2 diabetes mellitus (T2DM) [28]. In contrast, the alleles associated with the increased LDL cholesterol may reduce the risk of T2DM. Regarding the fact that PCSK9 is the main determinant factor in regulating plasma LDL cholesterol level and the genetic variants of PCSK9 may predispose to the incidence of T2DM, this study investigated the relationship between the PCSK9 rs615563 genotypes (a GOF allele) with the levels of circulating lipids and the incidence of diabetes on subsamples from a 5-year (2009-2014) cohort study. The current study results are significant because worldwide regulatory agencies have cleared PCSK9 inhibitors as cholesterol-lowering drugs.

\section{Main text \\ Methods \\ Study population}

The data reported in this study are based on subsamples from a 5-year (2009-2014) cohort study of the adult population of Ahvaz, Iran (590 subjects). The detailed information about the study population is described in Additional file 1.

\section{Biochemical analyses}

Fasting blood samples were drawn from the antecubital vein and then centrifuged at $2500 \mathrm{rpm}$ for $10 \mathrm{~min}$ to separate serum. The detailed procedures are described in Additional file 1.

\section{Genotyping of rs615563 variants}

The rs615563 was genotyped using polymerase chain reaction (PCR) -restriction fragment length polymorphism (RFLP). The amplified product of PCR was a $365 \mathrm{bp}$ fragment which was digested to three fragments of 32, 333, and $365 \mathrm{bp}$. The PCR products with homozygous allele (GG) were digested into two bands of 333 and $32 \mathrm{bp}$, while the heterozygous (GA) yielded three bands of 365,333 , and $32 \mathrm{bp}$, and finally, the homozygous allele
(AA) presented one band of $365 \mathrm{bp}$. The detailed procedure is described in Additional file 1.

\section{Statistical analysis}

Statistical analysis was performed with the SPSS, version 22. Quantitative data were reported as mean $\pm S D$ or median (interquartile range), and qualitative data were reported as frequency and percentage.

One-way ANOVA and Kruskal-Wallis tests were conducted for quantitative variables with normal distribution or non-normal distribution, respectively, to define the relationship between the variables with more than two groups. Cluster analysis (ANCOVA) was used to control the confounding factors in the groups. We performed logistic regression analysis with and without adjustment for age and sex and body mass index (BMI). Odds ratios (OR) were calculated for different genotypes with different outcomes. A non-conditional logistic regression model for odds ratio with confidence intervals was used to investigate the relationship between the genotypes and the disease to control the possible confounding factors. A p-value $<0.05$ was considered statistically significant.

\section{Results}

We assessed the association of PCSK9 rs615563 genotypes with the levels of FBS, BMI, lipid parameters, including total cholesterol, HDL cholesterol, LDL cholesterol, non-HDL cholesterol, triglyceride, and liver enzyme levels (AST and ALAT). The results of genetic analysis and the frequency of the rs615563 variants in 590 subjects showed that 328 subjects had homozygous GG genotype, 205 subjects had heterozygote genotype GT, and 57 subjects showed homozygous TT genotype. When we adjusted our results for age, sex, and BMI, we did not find any significant association between the PCSK9 rs615563 variants and our variables in non-diabetic and diabetic participants (Tables 1, 2).

In the case-control analysis, we did not find any significant association between the PCSK9 rs615563 and type 2 diabetes [GG OR (95\% CI) $1.78(0.79,4.44) ; \mathrm{p}=0.16$ ], [AA OR (95\% CI) $1.48(0.84,2.61) ; \mathrm{p}=0.17$ ] and the incidence of type 2 diabetes over the 5 years of followup [GG OR $(95 \% \mathrm{CI}) 1.55(0.85,4.39) ; \mathrm{p}=0.16]$, [AA OR (95\% CI) $1.72(0.84,2.61) ; \mathrm{p}=0.26]$ (Table 3).

\section{Discussion}

To our knowledge, this is the first study investigating the association of the PCSK9 rs615563 polymorphism and the incidence of diabetes. According to the results, there was no significant difference in the distribution of rs615563 alleles among non-diabetic and type 2 diabetic patients in the case-control group. There were no 
Table 1 Associations between the PCSK9 rs615563 variant and, FBS, lipid homeostasis variables and liver enzyme levels in 500 nondiabetic participants

\begin{tabular}{|c|c|c|c|c|c|c|}
\hline \multirow[t]{2}{*}{ Clinical data } & \multirow[t]{2}{*}{$\mathbf{N}$} & \multicolumn{3}{|c|}{ Mean/median data level by rs 615563 genotype } & \multirow[t]{2}{*}{$p$ value } & \multirow{2}{*}{$\begin{array}{l}\text { Adjusted } \\
\text { p-value }^{a}\end{array}$} \\
\hline & & GG & GA & AA & & \\
\hline $\operatorname{Sex}(M / F)$ & 197/303 & $\begin{array}{l}105 / 165 \\
38.9 / 61.1 \%\end{array}$ & $\begin{array}{l}67 / 116 \\
36.6 / 63.4 \%\end{array}$ & $\begin{array}{l}25 / 22 \\
53.2 / 46.8 \%\end{array}$ & - & - \\
\hline Age (years) & 499 & $41.52 \pm 14.97$ & $39.34 \pm 14.96$ & $40.09 \pm 12.37$ & 0.296 & - \\
\hline BMI $(\mathrm{kg} / \mathrm{m} 2)$ & 495 & $26.39 \pm 14.7$ & $27.1815 .62 \pm$ & $27.10 \pm 18.83$ & 0.380 & - \\
\hline $\mathrm{FBS}(\mathrm{mg} / \mathrm{dl})(\mathrm{IQR})$ & 500 & $92(85-100)$ & $92(85-98)$ & $92(82-99)$ & 0.649 & 0.72 \\
\hline $\mathrm{TC}(\mathrm{mg} / \mathrm{dL})$ & 500 & $184.07 \pm 38.04$ & $181.53 \pm 38.15$ & $185.80 \pm 36.97$ & 0.701 & 0.8 \\
\hline $\mathrm{HDL}-\mathrm{C}(\mathrm{mg} / \mathrm{dL})$ & 500 & $46.72 \pm 10.23$ & $46.77 \pm 9.09$ & $45.60 \pm 9.47$ & 0.745 & 0.96 \\
\hline Non-HDL (mg/dL) & 500 & $136.06 \pm 35.24$ & $133.18 \pm 35.59$ & $139.53 \pm 37.55$ & 0.488 & 0.66 \\
\hline LDL-C (mg/dL) & 500 & $107.70 \pm 33.45$ & $105.40 \pm 36.80$ & $108.27 \pm 13$ & 0.760 & 0.81 \\
\hline $\mathrm{TG}(\mathrm{mg} / \mathrm{dl})(\mathrm{IQR})$ & 500 & $115(78.5-163)$ & $103(72-146)$ & $119(72-195)$ & 0.137 & 0.36 \\
\hline ALT (U/I) (IQR) & 428 & $12(9-17.75)$ & $13(9-18)$ & $12(8-15)$ & 0.465 & 0.7 \\
\hline AST (U/I) (IQR) & 428 & $27.5(23-35)$ & & $28(23-34)$ & 0.894 & 0.64 \\
\hline
\end{tabular}

Data are expressed as mean \pm SD or median (IQR)

IQR interquartile range, FBS, Fasting blood sugar, $A L A T$ alanine aminotransferase, $A S T$ aspartate aminotransferase, $H D L-C H D L$ cholesterol, $L D L-C L D L-c h o l e s t e r o l, T C$ total cholesterol, TG triglyceride

${ }^{a}$ Adjusted for sex, age, BMI

Table 2 Associations between the PCSK9 rs615563 variant and, FBS, lipid homeostasis variables and liver enzyme levels in patients with diabetes

\begin{tabular}{|c|c|c|c|c|}
\hline \multirow[t]{2}{*}{ Variables } & \multicolumn{3}{|l|}{ Genotype } & \multirow[t]{2}{*}{$p$-value ${ }^{a}$} \\
\hline & $\mathrm{GG}(n=58)$ & $\mathrm{GA}(\mathrm{n}=22)$ & $A A(n=10)$ & \\
\hline $\operatorname{Sex}(M / F)$ & $24 / 34$ & $13 / 9$ & $5 / 5$ & 0.35 \\
\hline Age (years) & $56.5 \pm 13$ & $54.36 \pm 10.5$ & $53.5 \pm 13$ & 0.57 \\
\hline $\mathrm{BMI}\left(\mathrm{kg} / \mathrm{m}^{2}\right)$ & $28 \pm 5$ & $26.82 \pm 4.5$ & $30.5 \pm 7$ & 0.32 \\
\hline $\mathrm{FBS}(\mathrm{mg} / \mathrm{dL})^{*}$ & $176(136 .-213)$. & 169.5 (134-193) & $158(128-177.5)$ & 0.2 \\
\hline $\mathrm{TC}(\mathrm{mg} / \mathrm{dL})^{*}$ & $199.87 \pm 41.41$ & $194.6 \pm 45.54$ & $198.95 \pm 38.95$ & 0.95 \\
\hline $\mathrm{TG}(\mathrm{mg} / \mathrm{dL})^{*}$ & $155(116-214)$ & 140 (92-246) & $162(124.25-193)$ & 0.46 \\
\hline $\mathrm{HDL}-\mathrm{C}(\mathrm{mg} / \mathrm{dL})^{*}$ & $45.8 \pm 8.84$ & $44.54 \pm 11$ & $46.3 \pm 9.8$ & 0.75 \\
\hline Non-HDL (mg/dL)* & $153.36 \pm 38.93$ & $152.72 \pm 37.92$ & $148.3 \pm 43.38$ & 0.7 \\
\hline $\mathrm{LDL}-\mathrm{C}(\mathrm{mg} / \mathrm{dL})^{*}$ & $117.65 \pm 39.40$ & $109.59 \pm 41.85$ & $118.70 \pm 52.03$ & 0.9 \\
\hline $\operatorname{ALAT}(\mathrm{U} / \mathrm{I})^{*}$ & $15(10-20)$ & $15(10-22.5)$ & $10(8-19)$ & 0.29 \\
\hline $\operatorname{AST}(\mathrm{U} / \mathrm{I})^{*}$ & $29(23-36)$ & $28.5(24-36.25)$ & $30(22.5-33.5)$ & 0.82 \\
\hline
\end{tabular}

adjust for sex, age, BMI

significant differences in FBS levels of homozygous and heterozygous subjects (Table 1 ).

In the case-control study, we showed that the incidence of type 2 diabetes in rs615563 variants did not increase during a five-year follow-up.

PCSK9 by degrading LDL receptor on the cells surface represents a crucial regulator of LDL receptor [2, 22, 33]. Targeting PCSK9 with monoclonal antibodies demonstrates the newest and most promising pharmacological tool for the treatment of hypercholesterolemia. Different common and rare gene variants influence
PCSK9 function. Its gain-of-function mutations have been associated with hypercholesterolemia, and loss-offunction mutations result in low LDL cholesterol levels $[1,10,31]$. PCSK9 rs615563, a gain of function mutation, is associated with elevated LDL cholesterol levels. In a study conducted by Tao Guo et al., positive correlations were observed between total plasma cholesterol and triglyceride, with PCSK9 rs615563 in patients with hyperlipidemia [18]. In another study, Guo et al. demonstrated an association of the rs615563 with increased triglyceride in two healthy Chinese populations [17]. In our study, we 
Table 3 Type 2 diabetes case-control and incidence analysis rs615563 (G/A)

\begin{tabular}{|c|c|c|c|c|}
\hline & Non-Diabetic & Diabetic & OR $(\% 95 \mathrm{Cl})^{\mathrm{a}}$ & p-value \\
\hline \multicolumn{5}{|c|}{ Type 2 diabetes case-control analysis $(n=590)$} \\
\hline GG & $270(82.3 \%)$ & $58(17.7 \%)$ & $1.78(0.79-4.44)$ & 0.16 \\
\hline GA & $183(89.3 \%)$ & $22(10.7 \%)$ & Reference & \\
\hline AA & $47(82.5 \%)$ & $10(17.5 \%)$ & $1.48(0.84-2.61)$ & 0.17 \\
\hline \multicolumn{5}{|c|}{ Allele frequency } \\
\hline G & $72.3 \%$ & $77 \%$ & & \\
\hline A & $27.7 \%$ & $23 \%$ & & \\
\hline \multicolumn{5}{|c|}{ Type 2 diabetes incidence analysis $(n=580)$} \\
\hline GG & $270(83.6 \%)$ & $53(16.4 \%)$ & $1.55(0.85-4.39)$ & 0.16 \\
\hline GA & $183(90.6 \%)$ & $19(9.4 \%)$ & Reference & \\
\hline AA & $47(85.5 \%)$ & $8(14.5 \%)$ & $1.72(0.84-2.61)$ & 0.26 \\
\hline \multicolumn{5}{|c|}{ Allele frequency } \\
\hline G & $72.3 \%$ & $78 \%$ & & \\
\hline A & $27.7 \%$ & $22 \%$ & & \\
\hline
\end{tabular}

${ }^{\mathrm{a}}$ OR from a logistic regression model adjusted for age, sex and BMI

did not find any associations between total cholesterol and triglyceride with PCSK9 rs615563. The differences in the genetic background may partly explain the reason for these discrepancies. Guo et al. included healthy individuals from two different populations, Han and Jing, from China in their study. While the Han population is the largest nationality in china, Jing is the smallest one and is a relatively conservative and isolated population with low genetic heterogeneity [17]. Therefore, genetic background and mutations in other lipid-related genes in this population were most probably different from our studied population. Furthermore, the phenotypic expression of the PCSK9 mutations is affected by several environmental factors, including dietary habits, physical activity, and lifestyles that are different between the populations.

Our results are consistent with a recent study by Zamarrón-Licona et al. in healthy individuals and subclinical atherosclerosis patients. No significant association between total plasma cholesterol and triglyceride, with PCSK9 rs615563, was found in this study, and interestingly, the rs615563 polymorphism in the control group was associated with a decreased risk of hypertriglyceridemia [32].

Genetic studies of the effects of gene variants in a wide range have shown a link between the gene variants with decreased LDL cholesterol and increased risk of type 2 diabetes $[15,25]$. According to these findings, patients with autosomal familial hypercholesterolemia caused by a mutation in the LDL receptor and apolipoprotein B receptor are diagnosed with type 2 diabetes $50 \%$ less than those without these mutations [3]. There are ample LDL receptors on the cell surface of pancreatic beta-cells that play a pivotal role in the uptake and homeostasis of islet cholesterol. Experimental evidence using PCSK9deficient mice revealed that elevated expression of LDL receptor is associated with a decrease in LDL cholesterol level in the circulation, impaired glucose tolerance, and pancreatic islet abnormalities [13]. In the PCSK9 deficiency state, excessive cholesterol accumulation affects pancreatic beta-cell function that may reduce its ability to secrete insulin in response to glucose [13]. These findings were further advocated in subjects with a PCSK9 loss of function. In line with this evidence, Mendelian randomization studies also confirmed the association of PCSK9 variation with the increased risk of type 2 diabetes [29].

In contrast to these findings, we did not find an association between PCSK9 rs615563 genotypes and the diabetic status or its incidence in the current study. Although, as noted above, some studies suggest a possible association between PCSK9 genetic variants and an increased risk of type 2 diabetes, there are also studies with contradictory findings. For example, a study has reported pharmacological treatments with PCSK9 inhibitors in patients with primary hypercholesterolemia resulted in increased fasting glucose levels compared to placebo but with no increase in the incidence of diabetes [14]. There is also some discrepant evidence regarding the role of PCSK9 in glucose homeostasis obtained from the studies using PCSK9-deficient mice. In one of these studies conducted by Langhi $\mathrm{C}$ et al., they found that PCSK9 deficiency does not affect glucose-stimulated insulin secretion in mouse islets [24], while in the study conducted by Mbikay M et al., PCSK9-null mice showed hyperglycemia and signs of apoptosis in their pancreatic islets [26].

Furthermore, a study conducted by Saavedra et al. found that in patients with autosomal familial hypercholesterolemia, the presence of PCSK9 InsLEU variant (a loss-of-function mutation) was associated with lower LDL cholesterol. However, the prevalence of diabetes and pre-diabetes in these people was twice as high as those without this polymorphism [27]. In contrast, in a study by Bonnefond A et al., the PCSK9 P.R46L variant (the loss-of-function genetic variant) was not associated with impaired glucose homeostasis. These researchers showed that in individuals with the P.R46L variant, the incidence of type 2 diabetes after nine years follow-up or the risk of type 2 diabetes did not increase. They found no relationship between the distribution of genotypic P.R46L variants and fasting glucose, $\mathrm{HbA1C}$, insulin resistance markers (HOMA-IR), or insulin secretion (HOMA-B) [4]. The reasons for these discrepancies are unclear, but the concentration and duration of PCSK9 deficiency may contribute to the increased risk of diabetes and need to be addressed in the future. 


\section{Limitations}

There are some limitations in our study to be considered. In the present study, we only focused on the effects of rs615563 variants, the most frequent PCSK9 GOF, while other rare GOF variants of PCSK9 may affect glucose homeostasis. We also did not evaluate the level of PCSK9 and the relationship between circulating PCSK9 and diabetes in the population.

\begin{abstract}
Abbreviations
PCSK9: Proprotein convertase subtilisin/kexin type 9; PCR: Polymerase chain reaction; RFLP: Restriction fragment length polymorphism; NARC1: Neural apoptosis-regulated convertase 1; LDL: Low-density lipoprotein; LDLR: LDL receptor; GOF: Gain-of-function; LOF: Loss-of-function; HDL-C: Highdensity lipoprotein cholesterol; AST: Aspartate transaminase; ALAT: Alanine aminotransferase; HOMA-IR: Homeostatic Model Assessment for Insulin Resistance; HOMA-B: The HOMA-beta cell function; HbA1c: Hemoglobin A1c.
\end{abstract}

\section{Supplementary Information}

The online version contains supplementary material available at https://doi. org/10.1186/s13104-021-05723-4.

Additional file 1: Details of the study population, biochemical analyses, genotyping of rs615563 variants of the methods section.

\section{Acknowledgements}

This paper is issued from the research project with the registered number of D-9509. Financial support was provided by the Health Research Institute, Diabetes Research Center, Ahvaz Jundishapur University of Medical Sciences.

\section{Authors' contributions}

NM designed the study. STJ performed all assays. BC analyses the data. MN and MTJ contributed to interpreting the results. SJT wrote the first draft. MN and MTJ revised the manuscript. All authors read and approved the final manuscript.

\section{Funding}

This study was supported by the Ahvaz Jundishapur University of Medical Sciences (Grant No. D-9509). The funding source has no role in the study's design, analysis, interpretation of data, and publication of the manuscript.

\section{Availability of data and materials}

The datasets used and/or analyzed during the current study are available from the corresponding author on reasonable request.

\section{Declarations}

Ethics approval and consent to participate

Our study was approved by the Ethics Committee of Ahvaz Jundishapur University of Medical Sciences.

\section{Consent for publication}

Not applicable.

\section{Competing interests}

The authors declare that they have no competing interests.

\section{Author details}

${ }^{1}$ Health Research Institute, Diabetes Research Center, Ahvaz Jundishapur University of Medical Sciences, Ahvaz, Iran. ${ }^{2}$ Department of Laboratory Sciences, Faculty of Paramedicine, Ahvaz Jundishapur University of Medical Sciences, Ahvaz, Iran. ${ }^{3}$ Department of Epidemiology and Biostatistics, School of Health, Ahvaz Jundishapur University of Medical Sciences, Ahvaz, Iran.
${ }^{4}$ Hyperlipidemia Research Center, Ahvaz Jundishapur University of Medical Sciences, Ahvaz, Iran.

Received: 1 March 2021 Accepted: 29 July 2021

Published online: 11 August 2021

\section{References}

1. Abifadel M, Varret M, Rabès JP, Allard D, Ouguerram K, et al. Mutations in PCSK9 cause autosomal dominant hypercholesterolemia. Nat Genet. 2003;34:154-6.

2. Benjannet S, Rhainds D, Essalmani R, Mayne J, Wickham L, et al. NARC-1/ PCSK9 and its natural mutants: zymogen cleavage and effects on the low density lipoprotein (LDL) receptor and LDL cholesterol. J Biol Chem. 2004;279:48865-75.

3. Besseling J, Kastelein JJ, Defesche JC, Hutten BA, Hovingh GK. Association between familial hypercholesterolemia and prevalence of type 2 diabetes mellitus. JAMA. 2015;313:1029-36.

4. Bonnefond A, Yengo L, Le May C, Fumeron F, Marre M, et al. The loss-offunction PCSK9 p. R46L genetic variant does not alter glucose homeostasis. Diabetologia. 2015;58:2051-5.

5. Brouwers MC, Troutt JS, van Greevenbroek MM, Ferreira I, Feskens EJ, et al. Plasma proprotein convertase subtilisin kexin type 9 is not altered in subjects with impaired glucose metabolism and type 2 diabetes mellitus, but its relationship with non-HDL cholesterol and apolipoprotein B may be modified by type 2 diabetes mellitus: the CODAM study. Atherosclerosis. 2011;217:263-7.

6. Brown MS, Goldstein JL. Biomedicine. Lowering LDL-not only how low, but how long? Science. 2006;311:1721-3.

7. Cariou B, Le Bras M, Langhi C, Le May C, Guyomarc'h-Delasalle B, et al. Association between plasma PCSK9 and gamma-glutamyl transferase levels in diabetic patients. Atherosclerosis. 2010;211:700-2.

8. Cariou B, Le May C, Costet P. Clinical aspects of PCSK9. Atherosclerosis. 2011;216:258-65.

9. Caselli C, Turco SD, Ragusa R, Lorenzoni V, Graaf MDd, et al. Association of PCSK9 plasma levels with metabolic patterns and coronary atherosclerosis in patients with stable angina. Cardiovasc Diabetol. 2019;18(1):1-12.

10. Cohen J, Pertsemlidis A, Kotowski IK, Graham R, Garcia CK, Hobbs HH. Low LDL cholesterol in individuals of African descent resulting from frequent nonsense mutations in PCSK9. Nat Genet. 2005;37(2):161-5.

11. Cohen JC, Boerwinkle E, Mosley TH Jr, Hobbs HH. Sequence variations in PCSK9, low LDL, and protection against coronary heart disease. N Engl J Med. 2006:354:1264-72.

12. Colhoun HM, Robinson JG, Farnier M, Cariou B, Blom D, et al. Efficacy and safety of alirocumab, a fully human PCSK9 monoclonal antibody, in high cardiovascular risk patients with poorly controlled hypercholesterolemia on maximally tolerated doses of statins: rationale and design of the ODYSSEY COMBO I and II trials. BMC Cardiovasc Disord. 2014;14:121.

13. Da Dalt L, Ruscica M, Bonacina F, Balzarotti G, Dhyani A, et al. PCSK9 deficiency reduces insulin secretion and promotes glucose intolerance: the role of the low-density lipoprotein receptor. Eur Heart J. 2019;40:357-68.

14. de Carvalho LSF, Campos AM, Sposito AC. Proprotein convertase subtilisin/kexin type 9 (PCSK9) inhibitors and incident type 2 diabetes: a systematic review and meta-analysis with over 96,000 patient-years. Diabetes Care. 2018;41:364-7.

15. Fall T, Xie W, Poon W, Yaghootkar H, Mägi R, et al. Using genetic variants to assess the relationship between circulating lipids and type 2 diabetes. Diabetes. 2015;64:2676-84.

16. Farnier M. PCSK9: from discovery to therapeutic applications. Arch Cardiovasc Dis. 2014;107:58-66.

17. Guo T, Yin RX, Huang F, Yao LM, Lin WX, Pan SL. Association between the DOCK7, PCSK9 and GALNT2 gene polymorphisms and serum lipid levels. Sci Rep. 2016;6:19079.

18. Guo T, Yin RX, Lin WX, Wang W, Huang F, Pan SL. Association of the variants and haplotypes in the DOCK7, PCSK9 and GALNT2 genes and the risk of hyperlipidaemia. J Cell Mol Med. 2016;20:243-65.

19. Hallman DM, Srinivasan SR, Chen W, Boerwinkle E, Berenson GS. Relation of PCSK9 mutations to serum low-density lipoprotein cholesterol in childhood and adulthood (from The Bogalusa Heart Study). Am J Cardiol. 2007;100:69-72. 
20. Huang CC, Fornage M, Lloyd-Jones DM, Wei GS, Boerwinkle E, Liu K. Longitudinal association of PCSK9 sequence variations with low-density lipoprotein cholesterol levels: the Coronary Artery Risk Development in Young Adults Study. Circ Cardiovasc Genet. 2009;2:354-61.

21. Ibarretxe D, Girona J, Plana N, Cabré A, Ferré R, et al. Circulating PCSK9 in patients with type 2 diabetes and related metabolic disorders. Clin Investig Arterioscler. 2016;28:71-8

22. Lagace TA, Curtis DE, Garuti R, McNutt MC, Park SW, et al. Secreted PCSK9 decreases the number of LDL receptors in hepatocytes and in livers of parabiotic mice. J Clin Invest. 2006;116:2995-3005.

23. Lakoski SG, Lagace TA, Cohen JC, Horton JD, Hobbs HH. Genetic and metabolic determinants of plasma PCSK9 levels. J Clin Endocrinol Metab. 2009;94:2537-43.

24. Langhi C, Le May C, Gmyr V, Vandewalle B, Kerr-Conte J, Krempf M, Pattou F, et al. PCSK9 is expressed in pancreatic delta-cells and does not alter insulin secretion. Biochem Biophys Res Commun. 2009;390(4):1288-93.

25. Lotta LA, Sharp SJ, Burgess S, Perry JRB, Stewart ID, et al. Association between low-density lipoprotein cholesterol-lowering genetic variants and risk of type 2 diabetes: a meta-analysis. JAMA. 2016;316:1383-91.

26. Mbikay M, Sirois F, Mayne J, Wang GS, Chen A, et al. PCSK9-deficient mice exhibit impaired glucose tolerance and pancreatic islet abnormalities. FEBS Lett. 2010;584:701-6.

27. Saavedra YGL, Dufour R, Baass A. Familial hypercholesterolemia: PCSK9 InsLEU genetic variant and prediabetes/diabetes risk. J Clin Lipidol. 2015;9(6):786-93.e1.

28. Sattar N, Preiss D, Murray HM, Welsh P, Buckley BM, et al. Statins and risk of incident diabetes: a collaborative meta-analysis of randomised statin trials. Lancet. 2010;375:735-42.
29. Schmidt AF, Swerdlow DI, Holmes MV Patel RS, Fairhurst-Hunter Z et al. PCSK9 genetic variants and risk of type 2 diabetes: a mendelian randomisation study. Lancet Diabetes Endocrinol. 2017;5:97-105.

30. Seidah NG, Benjannet S, Wickham L, Marcinkiewicz J, Jasmin SB, et al. The secretory proprotein convertase neural apoptosis-regulated convertase 1 (NARC-1): liver regeneration and neuronal differentiation. Proc Natl Acad Sci U S A. 2003:100:928-33.

31. Urban D, Pöss J, Böhm M, Laufs U. Targeting the proprotein convertase subtilisin/kexin type 9 for the treatment of dyslipidemia and atherosclerosis. J Am Coll Cardiol. 2013;62:1401-8.

32. Zamarrón-Licona E, Rodríguez-Pérez JM, Posadas-Sánchez R, VargasAlarcón G, Baños-González MA, et al. Variants of PCSK9 gene are associated with subclinical atherosclerosis and cardiometabolic parameters in Mexicans. The GEA Project. Diagnostics (Basel). 2021;11(5):774.

33. Zhang DW, Lagace TA, Garuti R, Zhao Z, McDonald M, et al. Binding of proprotein convertase subtilisin/kexin type 9 to epidermal growth factorlike repeat A of low density lipoprotein receptor decreases receptor recycling and increases degradation. J Biol Chem. 2007;282:18602-12.

34. Zhao Z, Tuakli-Wosornu Y, Lagace TA, Kinch L, Grishin NV, et al. Molecular characterization of loss-of-function mutations in PCSK9 and identification of a compound heterozygote. Am J Hum Genet. 2006;79:514-23.

\section{Publisher's Note}

Springer Nature remains neutral with regard to jurisdictional claims in published maps and institutional affiliations.
Ready to submit your research? Choose BMC and benefit from:

- fast, convenient online submission

- thorough peer review by experienced researchers in your field

- rapid publication on acceptance

- support for research data, including large and complex data types

- gold Open Access which fosters wider collaboration and increased citations

- maximum visibility for your research: over $100 \mathrm{M}$ website views per year

At BMC, research is always in progress.

Learn more biomedcentral.com/submissions 